\title{
Thy-1 depletion and integrin $\beta 3$ upregulation-mediated PI3K-Akt- mTOR pathway activation inhibits lung fibroblast autophagy in lipopolysaccharide-induced pulmonary fibrosis
}

\author{
Hanxi Wan ${ }^{1} \cdot$ Tingting Xie ${ }^{1} \cdot$ Qiaoyi Xu ${ }^{1} \cdot$ Xiaoting $\mathrm{Hu}^{1} \cdot$ Shunpeng Xing ${ }^{1} \cdot \mathrm{Hao} \mathrm{Yang}^{2} \cdot$ Yuan $^{\mathrm{Gao}}{ }^{1} \cdot \mathrm{Zhengyu} \mathrm{He}^{1}$
}

Received: 13 February 2019 / Revised: 13 April 2019 / Accepted: 2 May 2019 / Published online: 27 June 2019

(c) The Author(s), under exclusive licence to United States and Canadian Academy of Pathology 2019

\begin{abstract}
Lipopolysaccharide (LPS)-induced autophagy inhibition in lung fibroblasts is closely associated with the activation of the phosphatidylinositol 3-kinase/protein kinase B/mammalian target of rapamycin (PI3K-Akt-mTOR) pathway. However, the underlying mechanism remains unknown. In this study, we demonstrated that LPS activated the PI3K-Akt-mTOR pathway and inhibited lung fibroblast autophagy by depleting thymocyte differentiation antigen-1 (Thy-1) and upregulating integrin $\beta 3$ (Itgb3). Challenge of the human lung fibroblast MRC-5 cell line with LPS resulted in significant upregulation of integrin $\beta 3$, activation of the PI3K-Akt-mTOR pathway and inhibition of autophagy, which could be abolished by integrin $\beta 3$ silencing by specific shRNA or treatment with the integrin $\beta 3$ inhibitor cilengitide. Meanwhile, LPS could inhibit Thy-1 expression accompanied with PI3K-Akt-mTOR pathway activation and lung fibroblast autophagy inhibition; these effects could be prevented by Thy- 1 overexpression. Meanwhile, Thy- 1 downregulation with Thy- 1 shRNA could mimic the effects of LPS, inducing the activation of PI3K-Akt-mTOR pathway and inhibiting lung fibroblast autophagy. Furthermore, protein immunoprecipitation analysis demonstrated that LPS reduced the binding of Thy- 1 to integrin $\beta 3$. Thy- 1 downregulation, integrin $\beta 3$ upregulation and autophagy inhibition were also detected in a mouse model of LPS-induced pulmonary fibrosis, which could be prohibited by intratracheal injection of Thy- 1 overexpressing adeno-associated virus (AAV) or intraperitoneal injection of the integrin $\beta 3$ inhibitor cilengitide. In conclusion, this study demonstrated that Thy- 1 depletion and integrin $\beta 3$ upregulation are involved in LPS-induced pulmonary fibrosis, and may serve as potential therapeutic targets for pulmonary fibrosis.
\end{abstract}

\section{Introduction}

Pulmonary fibrosis is a well-recognized feature of acute respiratory distress syndrome (ARDS) [1-3], and lipopolysaccharide (LPS) is a considerable factor causing sepsisassociated ARDS and pulmonary fibrosis [4-6]. However, the

These authors contributed equally: Hanxi Wan, Tingting Xie

Yuan Gao

rj_gaoyuan@163.com

$\triangle$ Zhengyu He

zhengyuheshsmu@163.com

1 Department of Critical Care Medicine, Renji Hospital, School of Medicine, Shanghai Jiaotong University, 200127 Shanghai, China

2 Department of Anesthesiology, Shanghai Pulmonary Hospital, Tongji University School of Medicine, 200433 Shanghai, China related mechanisms are not fully understood. Previous studies have shown that autophagy inhibition in lung tissues is closely associated with pulmonary fibrosis [7, 8]. In addition, our recent study revealed that LPS could inhibit lung fibroblast autophagy by activating the phosphatidylinositol-3-kinaseprotein kinase B-mammalian target of rapamycin (PI3K-AktmTOR) pathway [9]. However, the mechanism underlining LPS-induced PI3K-Akt-mTOR pathway activation and lung fibroblast autophagy inhibition remains largely unknown.

Thy-1 is a glycoprotein anchored by glycophosphatidylinositol (GPI) on the surface of fibroblasts [10]. Moderate expression of Thy- 1 was detected in lung fibroblasts of normal lung tissues [11]. However, reduced Thy-1 expression was reported to be associated with aberrant proliferation and activation of lung fibroblasts in LPS or bleomycin-induced pulmonary fibrosis $[12,13]$. Thy- 1 also binds to integrin $\beta 3$ (Itgb3) and inhibits skin dermal cell proliferation, and its depletion could induce the proliferation of skin dermal cells [14]. 
Integrins, which serve as a bridge between the extracellular matrix and the intracellular cytoskeleton, transduce a variety of signals from the extracellular matrix that affect cell survival and differentiation $[15,16]$. Integrin $\beta 3$ is the main integrin heterodimer on the surface of lung fibroblasts [17]. Previous studies have shown a protective role for integrin $\beta 3$ in lung disease, e.g., programmed cell death prevention in lung fibroblasts in response to transforming growth factor- $\beta 1$ (TGF- $\beta 1$ ) stimulation with activation of survival signals during TGF- $\beta 1$ stimulation [18]. However, little is known regarding the role of integrin $\beta 3$ in sepsisinduced pulmonary fibrosis. Multiple studies suggested that integrins are involved in the regulation of autophagy by activating its downstream PI3K-Akt signaling pathway, thereby controlling lung fibroblast viability and migration $[17,19]$. Therefore, we hypothesized that in LPS-induced pulmonary fibrosis, LPS may activate the PI3K-Akt-mTOR pathway and inhibit lung fibroblast autophagy by downregulating Thy- 1 and upregulating integrin $\beta 3$.

In this study, based on the cellular model of LPS-induced lung fibroblast autophagy inhibition and a mouse model of LPS-induced pulmonary fibrosis, we aimed to assess the role of Thy- 1 and integrin $\beta 3$ in the process of LPS-induced PI3K-Akt-mTOR pathway activation and lung fibroblast autophagy inhibition through genetic or pharmacological interventions.

\section{Material and methods}

\section{Ethics statement and animals}

Male C57BL/6 mice (8-week-old; 20-25 g) were obtained from Shanghai SLAC Laboratory Animal, China. Animals were housed under controlled temperature $\left(22-24^{\circ} \mathrm{C}\right)$ under a $12 \mathrm{~h} / 12 \mathrm{~h}$ light/dark cycle, with free access to food and tap water. All procedures in this study were carried out in accordance with the guidelines for animal care published by the United States' National Institutes of Health (NIH) for animal care (Guide for the Care and Use of Laboratory Animals, Department of Health and Human Services, NIH Publication No. 86-23, revised 1985). The study was approved by Renji hospital, Shanghai Jiao Tong University School of Medicine, Shanghai, China (Permit number: RJ20170930).

\section{Regents and antibodies}

Lipopolysaccharide (Escherichia coli O127:B8) was purchased from Sigma (USA). The integrin $\beta 3$ inhibitor cilengitide (\#S7077) was purchased from Selleckchem (USA). The primary antibodies used in this study were: rabbit anti-Thy-1 (ab225, Abcam, USA), mouse anti- integrin $\beta 3$ (sc-46655, Santa Cruz, USA), rabbit anti-LC3 I/ II (L7453, Sigma, USA), rabbit anti-p62/SQSTM1 (\#5114, Cell Signaling Technology, USA), rabbit anti-Akt (\#4691, Cell Signaling Technology, USA), rabbit anti-p-Akt (\#4060, Cell Signaling Technology, USA), rabbit antimTOR (\#2983, Cell Signaling Technology, USA), rabbit anti-p-mTOR (\#2971, Cell Signaling Technology, USA) and mouse anti-GAPDH (30201ES20, Yeasen, China). Goat anti-mouse (A0216, Beyotime, China) and goat antirabbit (A0208, Beyotime, China) secondary antibodies were used as well.

\section{Experimental design and treatment}

The human lung fibroblast MRC-5 cell line was purchased from the Cell Bank of the Chinese Academy of Sciences (Shanghai, China), and cultured in Minimum Essential Medium (MEM) (Gibco, Grand island, NY, USA) supplemented with $10 \%$ fetal bovine serum (FBS; Gibco), $100 \mathrm{U} /$ $\mathrm{ml}$ penicillin and $100 \mu \mathrm{g} / \mathrm{ml}$ streptomycin (Gibco), at $37^{\circ} \mathrm{C}$ in a humidified atmosphere of $5 \% \mathrm{CO}_{2}$ and $95 \%$ air.

MRC-5 cells in the logarithmic growth phase were seeded into 6-well plates at a density of $2 \times 10^{5}$ cells $/ \mathrm{mL}(2 \mathrm{~mL}$ in each well), and stimulated with $1 \mu \mathrm{g} / \mathrm{ml}$ LPS to generate an autophagy inhibition model. Then, treatment with cilengitide (\#S7077, Selleck, an integrin $\beta 3$ inhibitor), integrin $\beta 3$ knockdown lentivirus (Itgb3-KD), Thy-1 knockdown lentivirus (Thy-1-KD) and Thy-1 overexpression lentivirus (Thy-1-OE) (Genomeditech, Shanghai, China) were used to inhibit or overexpress integrin $\beta 3$ or Thy-1 at the protein and gene levels. Cells were collected after 6 and $24 \mathrm{~h}$, respectively, for the detection of signaling molecules and autophagy-associated proteins.

Animal experiments were carried out in C57/BL6 mice; $5 \mathrm{mg} / \mathrm{kg}$ LPS was intraperitoneally injected for 5 days to establish a pulmonary fibrosis model [20], and the integrin $\beta 3$ inhibitor cilengitide was intraperitoneally injected to inhibit integrin $\beta 3$, while Thy- 1 overexpression adenoassociated virus (AAV) was intratarsally injected to overexpress Thy-1. Lung tissue samples were collected 30 days after LPS stimulation.

\section{Lentivirus transfection}

MRC- 5 cells were seeded in a 12 -well plate at a density of $5 \times 10^{4}$ cells/well. Subsequently, lentivirus (Shanghai GeneChem, China) and polybrene $(5 \mu \mathrm{g} / \mathrm{ml})$ were added to each well. Transfection efficiency was assessed by green fluorescent protein (GFP) detection, and cells were selected with puromycin (CAS 58-58-2, Santa Cruz, USA) $(2 \mu \mathrm{g} / \mathrm{ml})$. Stable cell lines were established after a 1-week of selection. The expression levels of relevant proteins (Thy- 1 and integrin $\beta 3$ ) were examined by Western blot. 
The primers used were: Thy-1, F-5'-TCG CTCCTGCTAACAGTCT- $3^{\prime}$ and R-5'-AGACTGTTAG CAGGAGAGCGA-3'; integrin $\beta 3, \quad$ F-5'-GTGACCT GAAGGAGAATCTGC- $3^{\prime}$ and R-5'-CCGGAGTGCAA TCCTCTGG-3'.

\section{Thy-1 overexpression by AAV transfection}

The mouse Thy- 1 was cloned from the PGMThy- 1 plasmid by polymerase chain reaction (PCR) using the following primers: forward 5'-CCGGAATTCGCCACCATGAACCCAGCCATCAGCG-3' and reverse 5'- CCGGGATCCCAGAGAAATGAAGTCCAGGGCTTG-3'. All AAVs are purchased from Genomeditech (Shanghai, China).

AAV expressing vector or Thy-1 was delivered to the mouse lung by intratarsal injection in $50 \mu \mathrm{l}$ PBS containing $5 \times 10^{10} \mu \mathrm{g}$ per mouse, and termed vector-AAV and Thy-1AAV mice, respectively.

\section{Western blot}

The protein samples used for western blot were extracted with RIPA lysis buffer (Beyotime Biotechnology, China) supplemented with protease inhibitors (Roche, China). Protein concentrations were determined with the bicinchoninic acid (BCA) assay kit (Thermo scientific, USA). Equal amounts of protein were separated by SDS-polyacrylamide gel electrophoresis, transferred onto polyvinyl fluoride membranes (Merck KGaA, Darmstadt, Germany), and incubated with primary (rabbit anti-Thy-1 (ab225, Abcam, US), mouse anti- integrin $\beta 3$ (sc-46655, Santa Cruz, USA), rabbit anti-LC3 I/II (L7453, Sigma, USA), rabbit anti-p62/ SQSTM1 (\#5114, Cell Signaling Technology, USA), rabbit anti-Akt (\#4691, Cell Signaling Technology, USA), rabbit anti-p-Akt (\#4060, Cell Signaling Technology, USA), rabbit anti-mTOR (\#2983, Cell Signaling Technology, USA), rabbit anti-p-mTOR (\#2971, Cell Signaling Technology, USA) and mouse anti-GAPDH (30201ES20, Yeasen, China) antibodies, respectively. This was followed by incubation with the appropriate secondary antibodies, including goat anti-mouse (A0216, Beyotime, China) and goat anti-rabbit (A0208, Beyotime, China) antibodies, respectively. Signals were detected using the ECL Plus Western blotting system kit (Beyotime Biotechnology, China); band intensity was measured with the Image $\mathrm{Lab}^{\mathrm{TM}}$ software (Bio-Rad, USA).

\section{Transmission electron microscopy (TEM)}

A total of $1 \times 10^{5}$ MRC- 5 cells were fixed by treatment with fresh $2.5 \%$ glutaraldehyde at $4{ }^{\circ} \mathrm{C}$ for at least $4 \mathrm{~h}$, post-fixed with $1 \%$ osmium tetroxide for $1 \mathrm{~h}$, dehydrated in an ethanol gradient, embedded, and incubated respectively at 37, 45, and $60^{\circ} \mathrm{C}$ for $24 \mathrm{~h}$. Ultrathin sections prepared with an ultra-cut E ultra-microtome (Leica, Wetzlar, Germany) were stained with uranyl acetate and lead citrate, and observed on a TECNAI 10 transmission electron microscopy system from FEI (Hillsboro, USA). The semi-quantification of the results was obtained by counting the numbers of autophagosomes and autolysosomes in at least six cells.

\section{Quantitative real-time PCR (qRT-PCR)}

To assess Thy-1 mRNA expression, total RNA was isolated from lung tissue samples with TRIzol regent (Invitrogen) according to the manufacturer's instructions. Complementary DNA synthesis was performed using Prime Script RT Master Mix (Takara, China), and real-time PCR was carried out on a Light Cycler 480 real-time PCR system (Roche, USA) using iTaq universal SYBR Green Supermix (Bio-Rad, Hercules, CA, USA). The primers used for realtime PCR were: GAPDH, forward 5'-TGGTGAAGG TCGGTGTGAAC-3' and reverse 5'-GCTCCTGGAAGA TGGTGATGG-3'; Thy-1, forward 5'- CCGGAATTCGCC ACCATGAACCCAGCCATCAGCG-3' and reverse 5'- CC GGGATCCCAGAGAAATGAAGTCCAGGGCTTG -3'.

The $2^{-\Delta \Delta \mathrm{Ct}}$ method was employed to assess relative expression levels. GAPDH was used as an endogenous control.

\section{Immunofluorescence}

A total of $1 \times 10^{5}$ MRC- 5 cells cultured on 12 -well chamber slides were fixed with $4 \%$ paraformaldehyde and permeabilized with $0.1 \%$ Triton X-100. The slides were then blocked with $10 \%$ goat serum at room temperature for 10 min. Subsequently, the samples were incubated with primary antibodies, including rabbit anti-Thy-1 (ab225, Abcam, US) and mouse anti- integrin $\beta 3$ (sc-46655, Santa Cruz, USA), respectively, at $4{ }^{\circ} \mathrm{C}$ overnight, followed by incubation with Alexa Flour 488 or Cy3 secondary antibodies (Beyotime, China) for $1 \mathrm{~h}$ at room temperature. The slides were counterstained with DAPI and examined by fluorescence microscopy (Leica, Heidelberg, Germany). Primary antibodies included rabbit anti-Thy- 1 from CST and mouse anti-integrin $\beta 3$ from Santa Cruz.

\section{Co-Immunoprecipitation}

Co-immunoprecipitation was performed according to a previous report. ${ }^{16}$ Briefly, proteins were extracted with cell lysis buffer (KGP701 KGP701-100, KeyGEN BioTECH, China) with 1\% PMSF (KGP610, KeyGEN BioTECH, China). A total of $500 \mu \mathrm{g}$ of protein extract was then incubated with $2 \mu \mathrm{g}$ of appropriate immunoprecipitation (IP) antibodies, i.e., rabbit anti-Thy-1 (ab225, Abcam, US) or 
Fig. 1 LPS-induced autophagy inhibition is accompanied with Thy-1 downregulation and integrin $\beta 3$ upregulation in LPSinduced pulmonary fibrosis. The severity of pulmonary fibrosis was determined by hematoxylineosin (H\&E) staining; collagen deposition was revealed by MASSON staining (magnification, $\times 200$ ) (a). The expression of $\alpha$-SMA in lung tissues was also measured by Western blot (b). Collagen deposition was measured by hydroxyproline content and collagen level assessments (c). Representative Western blot images showing expression of Thy-1, integrin $\beta 3$, LC3 and P62 in the lung tissue (d). Values are mean $\pm \mathrm{SD}(n=6) . * p<0.05$ vs control group; $* * P<0.01 \mathrm{vs}$ control group
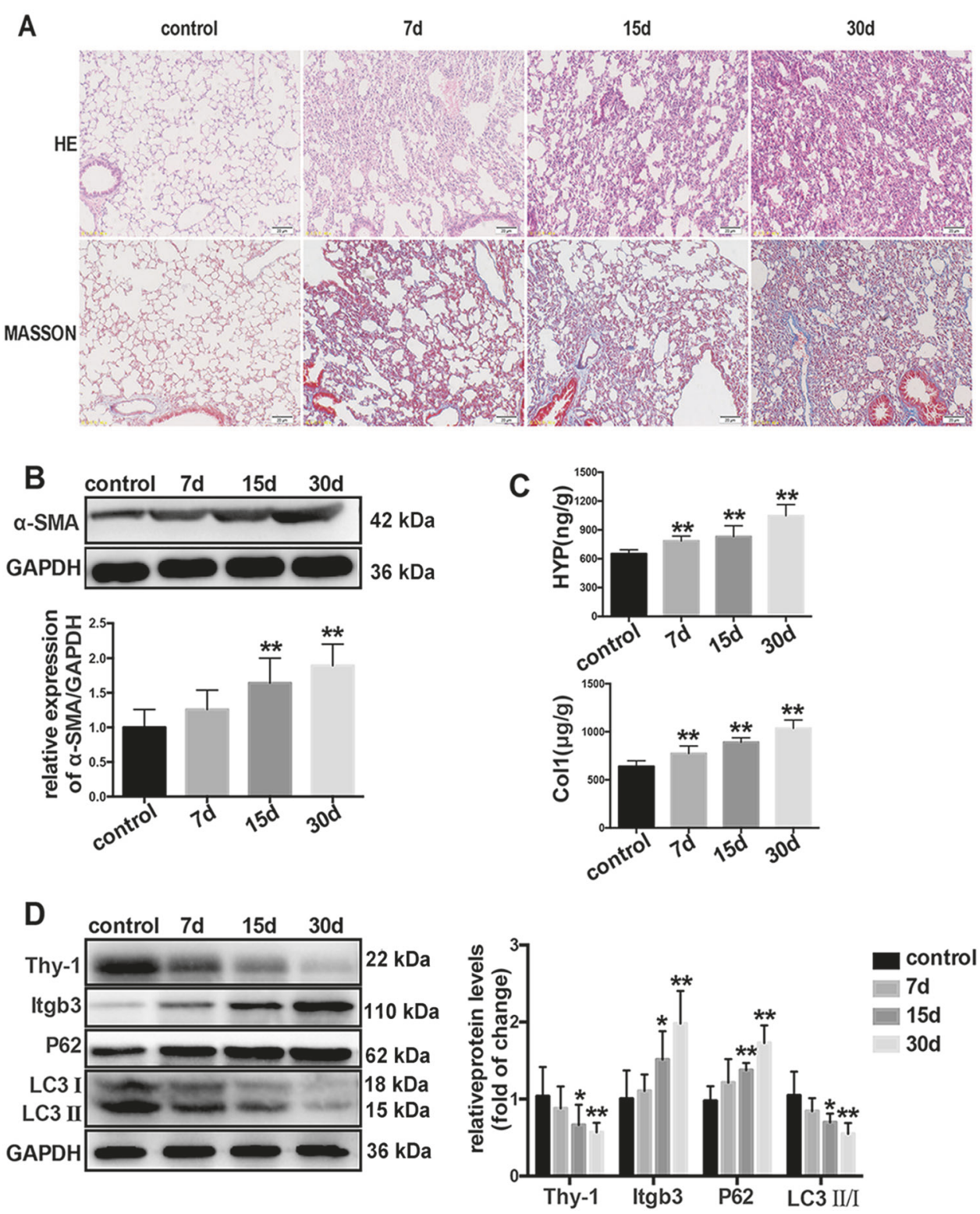

mouse anti- integrin $\beta 3$ (sc-46655, Santa Cruz, USA) at $4{ }^{\circ} \mathrm{C}$ overnight with shaking. Then, $20 \mu \mathrm{l}$ of protein A/G plus agarose (Thermo) was added, followed by incubation at $4{ }^{\circ} \mathrm{C}$ for $3 \mathrm{~h}$ with shaking. The pellets were washed five times with cell lysis buffer for $2 \mathrm{~min}$ and resuspended in 40 $\mu \mathrm{l}$ of $2 \times$ electrophoresis loading buffer. After boiling for 10 min, $20 \mu \mathrm{l}$ samples were used for SDS-PAGE and assessed by Western blot as described above.

\section{Flow cytometry}

PAC-1 binding to activated integrin $\beta 3$ was measured by flow cytometry (BD FACSVerse ${ }^{\mathrm{TM}}$, USA) using a modification of the technique previously described [15]. Briefly, $5 \times 10^{8}$ to $1 \times 10^{9} / \mathrm{ml}$ of MRC- 5 cells were incubated for 30 min with FITC mouse anti-human PAC-1 (340507, BD Biosciences, USA) $(20 \mu \mathrm{l})$ at room temperature. After washing with PBS, the cells were analyzed by flow cytometry; data were analyzed with the Flowjo (version 10) software.

\section{Hematoxylin and eosin (H\&E) and Masson's trichrome staining}

Pulmonary tissue samples were fixed by inflation with $4 \%$ paraformaldehyde overnight, dehydrated in $70 \%$ ethanol and embedded in paraffin wax. Sections of $5 \mu \mathrm{m}$ thickness were prepared and subjected to $H \& E$ and Masson's trichrome staining, respectively, as previously described [21]. 

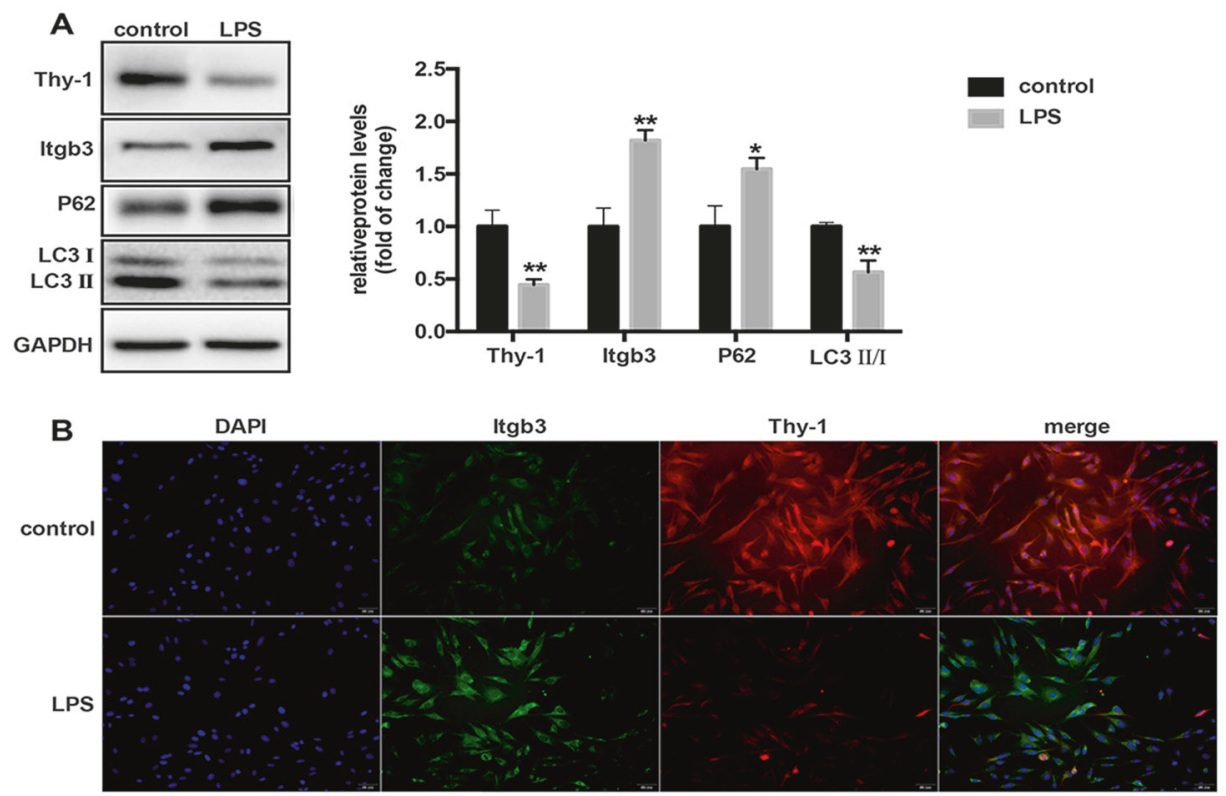

C

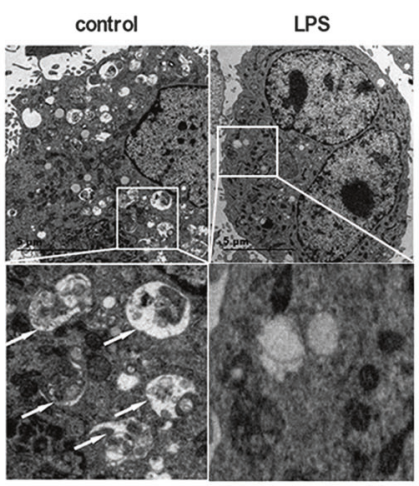

D
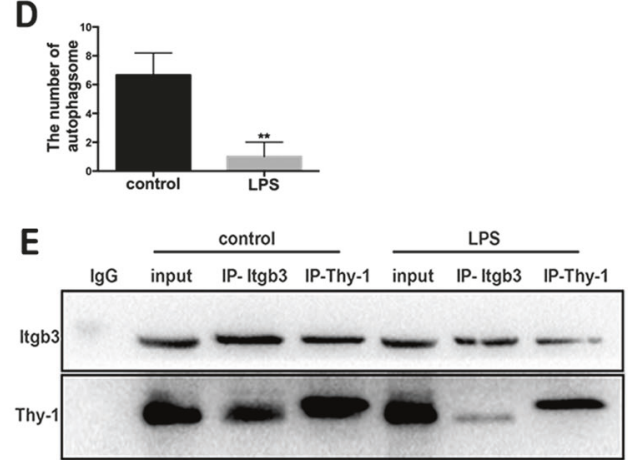

F
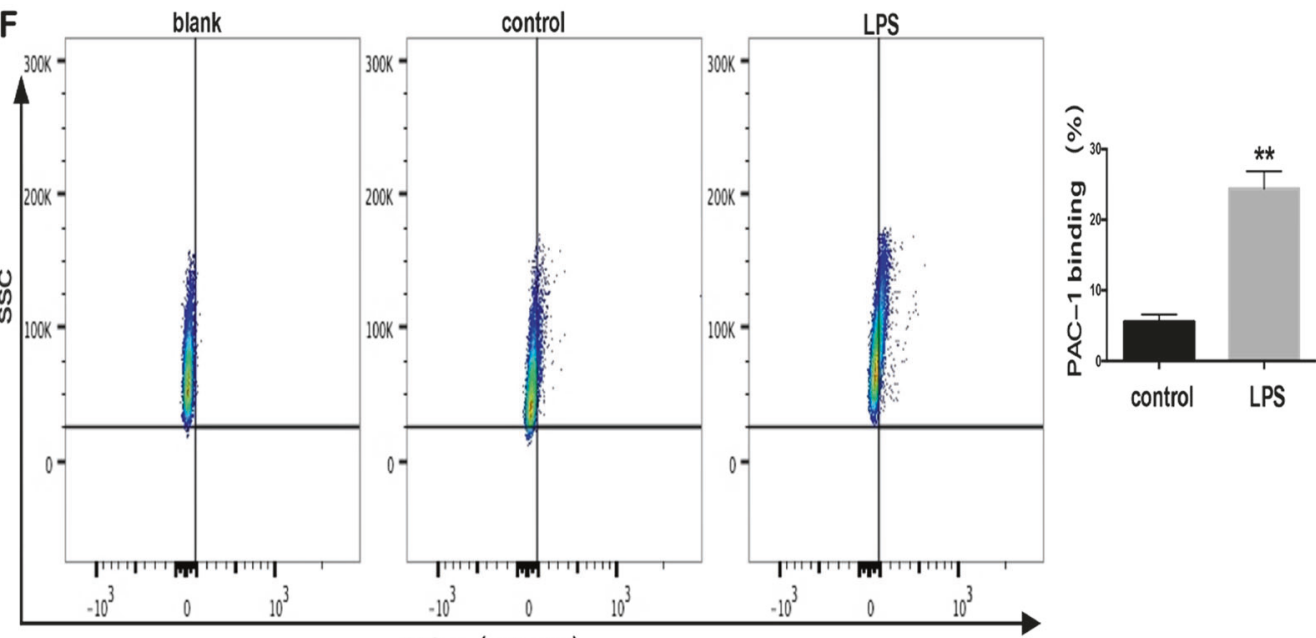

\section{Immunohistochemistry}

The levels of $\alpha$-SMA in the lung were assessed by IHC. Lung tissue sections were deparaffinized and incubated with
$1 \%$ albumin solution containing $0.1 \%$ Triton $\mathrm{X}-100$ at room temperature for $1 \mathrm{~h}$. The slices were then blocked with $3 \%$ normal goat serum containing $0.1 \%$ Triton $\mathrm{X}-100$ at room temperature for $1 \mathrm{~h}$. Next, the samples were sequentially 
Fig. 2 LPS-induced autophagy inhibition is accompanied with Thy-1 downregulation and integrin $\beta 3$ upregulation and activation in lung fibroblasts. Western blot was performed to detect Thy-1 and integrin $\beta 3$ in lung fibroblasts challenged with $1 \mu \mathrm{g} / \mathrm{ml}$ LPS for $6 \mathrm{~h}$, and P62 and LC3 in lung fibroblasts challenged with $1 \mu \mathrm{g} / \mathrm{ml}$ LPS for $24 \mathrm{~h}$ (a). Immunofluorescent staining of the nucleus (blue), integrin $\beta 3$ (green) and Thy-1 (red) in lung fibroblasts cultured in the absence or presence of $1 \mu \mathrm{g} / \mathrm{ml}$ LPS for $6 \mathrm{~h}(\mathbf{b})$. Lung fibroblasts were observed by transmission electron microscopy. White arrows indicate autophagosomes (c), the semi-quantification of autophagosomes of per cell were also shown (d). Co-IP was used to detect the interaction between Thy-1 and integrin $\beta 3$ in MRC-5 cells after LPS challenge. Western blot for coimmunoprecipitates of IgG control, input, Thy-1 and integrin $\beta 3$ (e). Flow cytometry was used to detect the expression of PAC-1, an integrin $\beta 3$ activation marker (f).Values are mean \pm SD from triplicate experiments. $* p<0.05, * * p<0.01$

incubated with rabbit anti- $\alpha$-SMA primary (1/2000) and goat anti-rabbit IgG secondary (1/1000) antibodies, each for $1 \mathrm{~h}$.

\section{Pulmonary Hydroxyproline (HYP) and collagen measurement}

To estimate total collagen deposition, an indicator of pulmonary fibrosis, the hydroxyproline content of the middle lobe of the left lung was measured with a commercially available hydroxyproline detection kit (Nanjing Jiancheng Bioengineering; Nanjing, China) according to the manufacturer's instructions. The collagen content of the upper lobe of the right lung was measured according to the protocol of a commercial collagen detection kit (Nanjing Jiancheng Bioengineering; Nanjing, China).

\section{Statistical analysis}

Data were processed with the Graph Pad PRISM7 software (USA), and presented as mean \pm standard deviation (SD). Two-tailed Student's $t$ test was used to compare two groups. Multiple groups were compared by one-way analysis of variance (ANOVA). $P<0.05$ was considered statistically significant.

\section{Results}

\section{LPS-induced autophagy inhibition is accompanied with Thy-1 downregulation and integrin $\beta 3$ upregulation in LPS-induced pulmonary fibrosis in vivo}

To investigate the time-course of LPS-induced pulmonary fibrosis in vivo, mice were intraperitoneally injected with saline or LPS $(5 \mathrm{mg} / \mathrm{kg}$ ) for 5 consecutive days, and samples were collected at day 7, 15 and 30 after LPS injection, respectively. As shown in Fig. 1a, mild pulmonary fibrosis was detected at 7 and 15 days after LPS treatment, and typical pulmonary fibrosis was observed at 30 days, accompanied with $\alpha$-SMA upregulation and increased hydroxyproline and collagen contents (Fig. 1b, c).

In addition, LPS also suppressed autophagy by upregulating P62 and decreasing the LC3II-to-LC3 I ratio, accompanied with decreased expression of Thy-1 and increased amounts of integrin $\beta 3$ in the lung tissue (Fig. 1d).

\section{LPS-induced autophagy inhibition is accompanied with Thy-1 downregulation and integrin $\beta 3$ upregulation and activation in lung fibroblasts in vitro}

To explore the effects of LPS on autophagy inhibition and Thy- 1 and integrin $\beta 3$ expression levels in lung fibroblasts, MRC-5 cells were challenged by LPS $(1 \mu \mathrm{g} / \mathrm{ml})$ for $6 \mathrm{~h}$. Compared with the control group, LPS challenge significantly inhibited Thy-1 expression and increased integrin $\beta 3$ amounts in lung fibroblasts (Fig. 2a), which was further confirmed by immunofluorescence (Fig. 2b). Furthermore, autophagy in lung fibroblasts was significantly inhibited after treatment with $1 \mu \mathrm{g} / \mathrm{ml}$ LPS for $24 \mathrm{~h}$, as indicated by increased P62 amounts, decreased LC3 II-to-LC3 I ratios and reduced autophagosome amounts detected by TEM (Fig. 2a, c, d).

Next, the interaction between Thy- 1 and integrin $\beta 3$ in MRC-5 cells was assessed by co-immunoprecipitation. After treating lung fibroblasts with LPS, the interaction between Thy- 1 and integrin $\beta 3$ was reduced (Fig. 2e). Then, flow cytometry was used to detect the expression of PAC-1, a marker of integrin $\beta 3$ activation, in lung fibroblasts. PAC1 expression was increased dramatically, which indicated integrin $\beta 3$ activation by LPS in MRC- 5 cells (Fig. 2f).

\section{Integrin $\beta 3$ inhibition precludes LPS-induced PI3K- Akt-mTOR activation and lung fibroblast autophagy inhibition}

Our previous study showed that LPS activates the PI3KAkt-mTOR pathway and inhibits lung fibroblast autophagy. To assess whether integrin $\beta 3$ is essential in LPS-induced PI3K-Akt-mTOR pathway activation and lung fibroblast autophagy inhibition, cilengitide, an inhibitor for integrin $\beta 3$, was applied. Western blot showed that integrin $\beta 3$ protein expression in lung fibroblasts was increased significantly $6 \mathrm{~h}$ after LPS challenge, and this effect was prohibited by cilengitide treatment (Fig. 3a, d). Meanwhile, activation of the PI3K-Akt-mTOR pathway was also detected by increased amounts of phosphorylated Akt ( $\mathrm{p}$ Akt) and phosphorylated mTOR (p-mTOR) $6 \mathrm{~h}$ after LPS challenge. However, no differences were observed after 

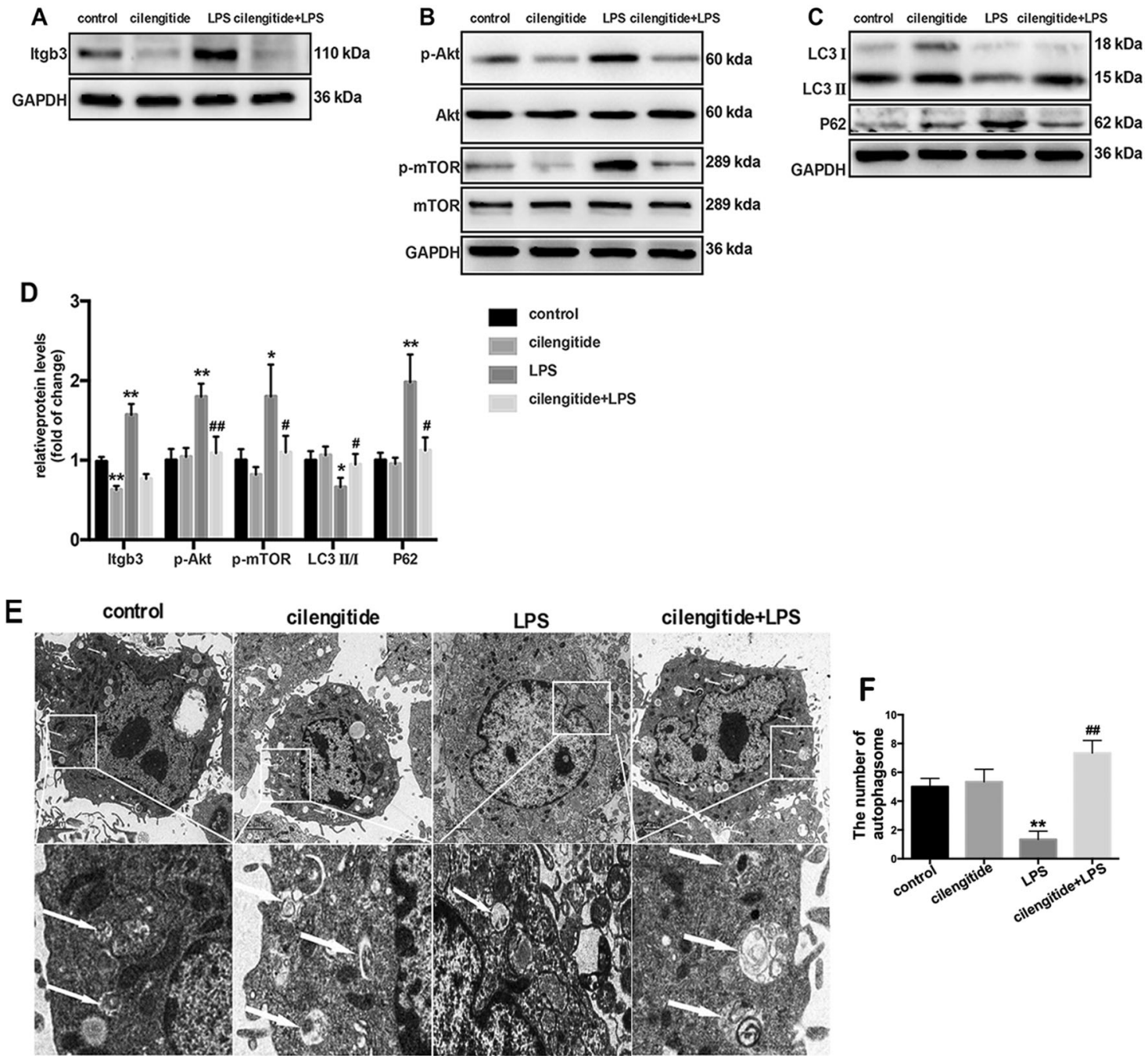

Fig. 3 Integrin $\beta 3$ inhibition precludes LPS-induced PI3K-Akt-mTOR activation and lung fibroblast autophagy blockade. Western blot was used to detect the expression of integrin $\beta 3$ (a), phospho-AKT (pAKT), total AKT, phospho-mTOR (p-mTOR) and total mTOR (b). Representative images showing protein expression of LC3 I, LC3 II and P62 in MRC-5 cells challenged without or with $1 \mu \mathrm{g} / \mathrm{ml}$ LPS in the absence or presence of the inhibitor cilengitide $(2 \mathrm{mM})$ for $24 \mathrm{~h}(\mathbf{c})$.
Quantitation of p-AKT/total AKT, p-mTOR/total mTOR, LC3 II/ I, integrin $\beta 3$ and P62 protein levels normalized to GAPDH (d). Lung fibroblasts were assessed by transmission electron microscopy. White arrows indicate autophagosomes (e), the semi-quantification of autophagosomes of per cell were also shown (f). Values are mean \pm SD from triplicate experiments. ${ }^{*} p<0.05$ vs control group; $* * p<0.01$ vs control group; ${ }^{\#} p<0.05$ vs LPS group; ${ }^{\# \#} p<0.05$ vs. LPS group cilengitide treatment (Fig. 3b, d). In addition, pretreatment with cilengitide increased the LC3 II-to-LC3 I ratio and decreased p62 levels, suggesting that cilengitide partially avoided LPS-induced autophagy inhibition (Fig. 3c, d). TEM images also showed that autophagosomes reappeared after cilengitide treatment (Fig. 3e, f).

To further assess the role of integrin $\beta 3$ in the inhibition of LPS-induced lung fibroblast autophagy, integrin $\beta 3$ was knocked down in lung fibroblasts.

Similar with the effect of the integrin $\beta 3$ inhibitor cilengitide, Itgb3-KD transfection prohibited LPS-induced integrin $\beta 3$ expression (Fig. 4a, d), PI3K-Akt-mTOR pathway activation (Fig. 4b, d) and autophagy inhibition in lung fibroblasts (Fig. 4c-f). These findings indicated that LPS activated the PI3K-Akt-mTOR pathway and inhibited lung fibroblast autophagy by upregulating integrin $\beta 3$.

\section{Thy-1 downregulation induces PI3K-Akt-mTOR activation and lung fibroblast autophagy inhibition}

To determine the effect of Thy-1 depletion on autophagy inhibition in lung fibroblasts, Thy-1 was knocked down in lung fibroblasts by transfection of Thy-1 shRNA. Thy-1 expression in lung fibroblasts was reduced significantly $6 \mathrm{~h}$ after LPS challenge or Thy-1 shRNA transfection (Fig. 5a, d). Thy-1 downregulation mimicked the effects of LPS, inducing PI3K-Akt-mTOR pathway activation and inhibiting lung fibroblast autophagy, as demonstrated 

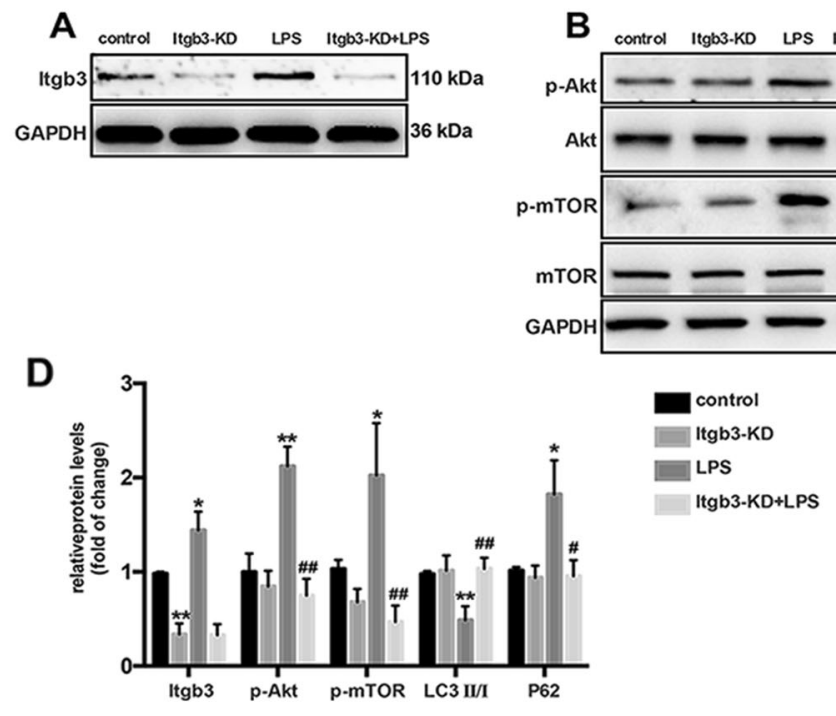

E

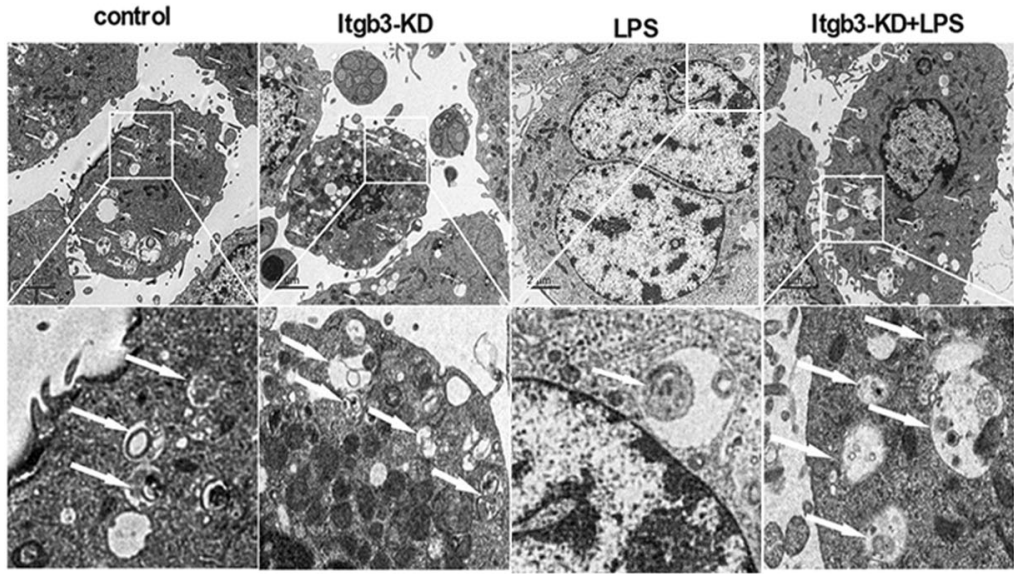

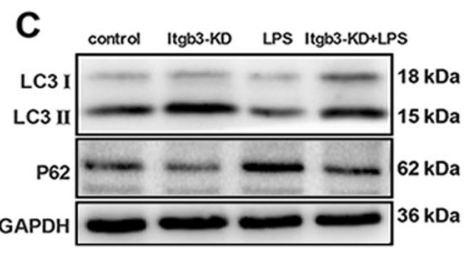

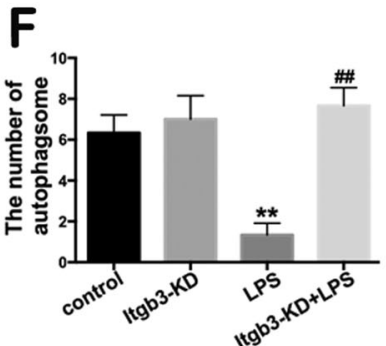

Fig. 4 Genetic integrin $\beta 3$ inhibition prevents LPS-induced PI3K-AktmTOR activation and lung fibroblast autophagy inhibition. Western blot was used to detect the expression levels of integrin $\beta 3$ (a), phospho-AKT (p-AKT), total AKT, phospho-mTOR (p-mTOR) and total mTOR (b) in lung fibroblasts after $6 \mathrm{~h}$ of $1 \mu \mathrm{g} / \mathrm{ml}$ LPS challenge after transfection with integrin $\beta 3$ shRNA or the empty vector. Representative images showing expression of LC3 I, LC3 II and P62 in lung fibroblasts after $24 \mathrm{~h}$ of $1 \mu \mathrm{g} / \mathrm{ml}$ LPS challenge after transfection with integrin $\beta 3$ shRNA or the empty vector (c). Quantification of p-AKT/total AKT, p-mTOR/total mTOR, LC3 II/ I, integrin $\beta 3$ and P62 protein levels normalized to GAPDH (d). Lung fibroblasts were observed by transmission electron microscopy. White arrows indicate autophagosomes (e), the semi-quantification of autophagosomes of per cell were also shown (f). Values are mean \pm SD from triplicate experiments. $* p<0.05$ vs. control group; $* * p<0.01$ vs. control group; ${ }^{\#} p<0.05$ vs. LPS group; ${ }^{\# \#} p<0.05$ vs. LPS group by increased expression levels of p-Akt and p-mTOR (Fig. 5b, d), reduced LC3 II-to-LC3 I ratios, increased expression of p62 (Fig. 5c, d) and reduced autophagosome amounts (Fig. 5e, f).

\section{Thy-1 overexpression precludes LPS-induced PI3K- Akt-mTOR activation and lung fibroblast autophagy inhibition}

To further assess whether Thy-1 depletion alters the effect of LPS, lung fibroblasts were transfected with Thy-1overexpression lentivirus (Thy-1-OE) to overexpress Thy-1. As shown in Fig. 6a and d, Thy-1-OE transfection prohibited LPS-induced Thy-1 depletion in lung fibroblasts, accompanied with a reversion of LPS-induced activation of the PI3K-Akt-mTOR pathway (Fig. 6b, d) and inhibition of lung fibroblast autophagy (Fig. 6c-f). These findings suggested that Thy-1 depletion mediated LPS-induced PI3KAkt-mTOR pathway activation and lung fibroblast autophagy inhibition.

\section{Thy-1 overexpression or integrin $\beta 3$ inhibition prevents LPS-induced autophagy inhibition and pulmonary fibrosis}

In order to investigate whether Thy-1 expression in vivo alters LPS-induced pulmonary fibrosis, Thy- $1^{+}$-AAV was transfected by intratracheal injection to overexpress the 

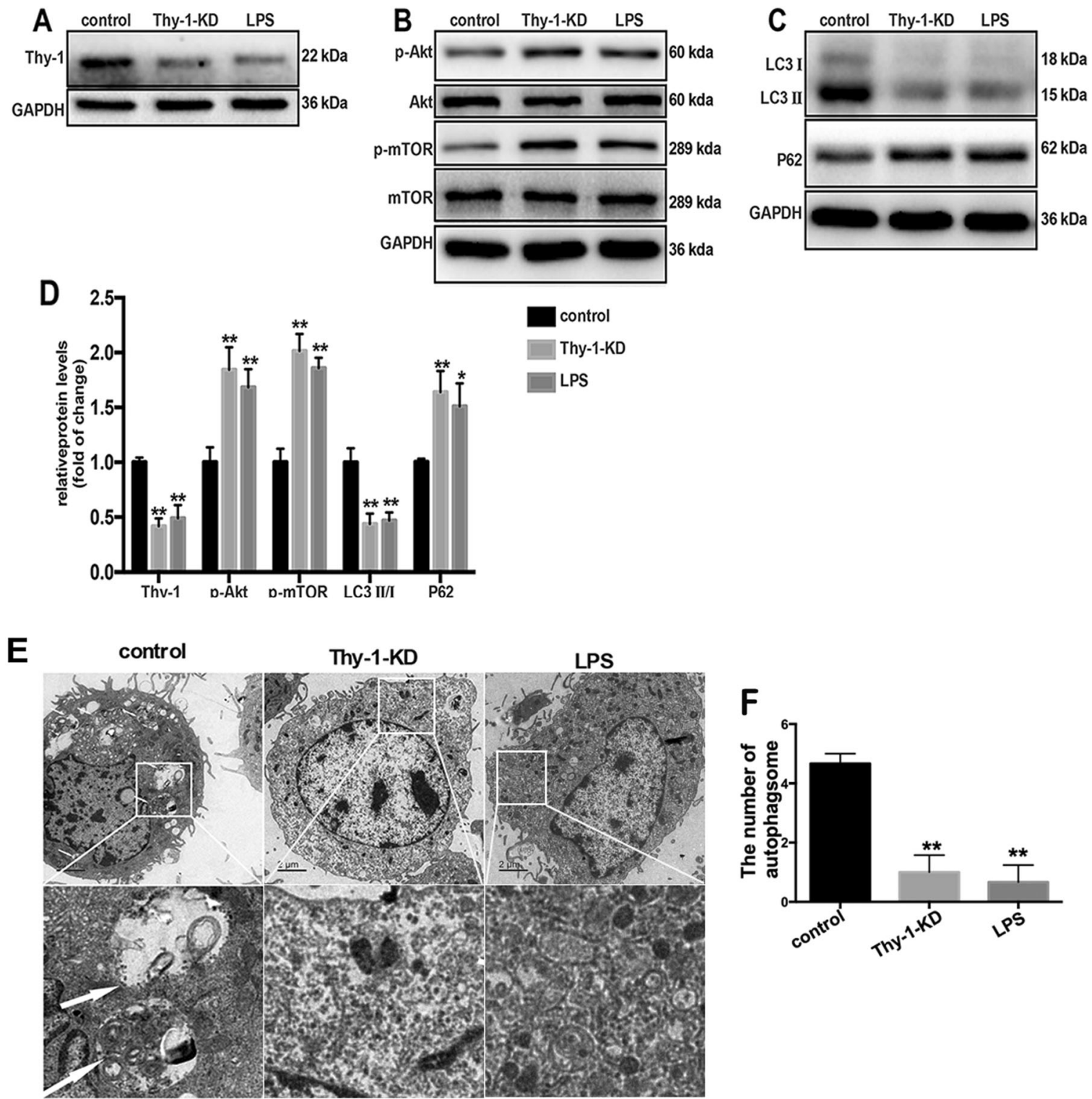

Fig. 5 Thy-1 downregulation induces PI3K-Akt-mTOR activation and lung fibroblast autophagy inhibition. Western blot was performed to detect the expression levels of Thy-1 (a), phospho-AKT (p-AKT), total AKT, phospho-mTOR (p-mTOR) and total mTOR (b) in lung fibroblasts after $6 \mathrm{~h}$ of $1 \mu \mathrm{g} / \mathrm{ml}$ LPS challenge after transfection with Thy-1 shRNA or the empty vector. Representative images showing expression of LC3 I, LC3 II and P62 in lung fibroblasts after $24 \mathrm{~h}$ of $1 \mu \mathrm{g} / \mathrm{ml}$

Thy-1 gene in the mouse lung tissue, as shown in Fig. 7a. The mRNA expression of Thy-1 was increased significantly after Thy- $1^{+}$-AAV transfection (Fig. 7b). As shown in Fig. 7c, Thy- $1^{+}$-AAV transfection inhibited LPS-induced Thy-1 depletion in the lung tissue, accompanied with a reversion of LPS-induced decrease of LC3 II-to-LC3 I ratio and increased expression of P62. In addition, western blot analysis of $\alpha$-SMA, hydroxyproline and collagen content measurements, pathological analysis by $\mathrm{H} \& \mathrm{E}$ or MASSON -staining, and $\alpha$-SMA
LPS challenge after transfection with Thy shRNA or the empty vector (c). Lung fibroblasts were observed by transmission electron microscopy. White arrows indicate autophagosomes (e), the semiquantification of autophagosomes of per cell were also shown (f). Values are mean \pm SD from triplicate experiments. ${ }^{*} p<0.05$ vs. control group; $* * p<0.01$ vs. control group

immunohistochemistry showed that Thy- $1^{+}$-AAV transfection prohibited LPS-induced pulmonary fibrosis (Fig. 7d-f). These findings suggested that Thy-1 depletion mediated LPS-induced lung tissue autophagy inhibition in LPS-induced pulmonary fibrosis.

To further investigate whether pharmacologically altering integrin $\beta 3$ expression and activation in vivo affects LPS-induced pulmonary fibrosis, integrin $\beta 3$ was inhibited in the mouse lung tissue by intraperitoneal injection of cilengitide. As shown in Fig. 8a, cilengitide 

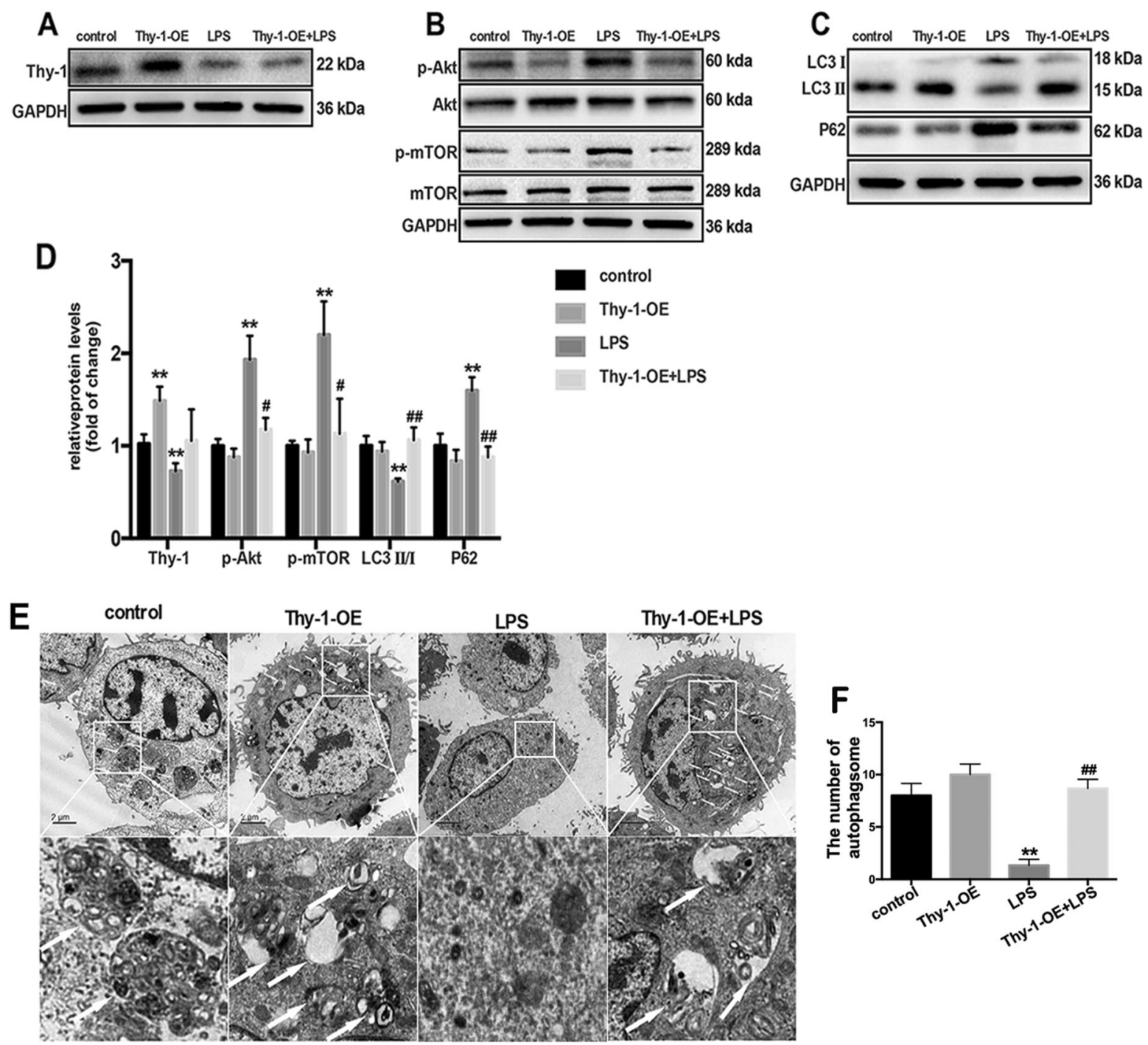

Fig. 6 Thy-1 overexpression precludes LPS-induced PI3K-Akt-mTOR activation and lung fibroblast autophagy inhibition. Western blot was performed to detect the expression levels of Thy-1(a), phospho-AKT (p-AKT), total AKT, phospho-mTOR (p-mTOR) and total mTOR (b) in lung fibroblasts after $6 \mathrm{~h}$ of $1 \mu \mathrm{g} / \mathrm{ml}$ LPS challenge after transfection with Thy-1-OE or the empty vector. Representative images showing expression of LC3 I, LC3 II and P62 in lung fibroblasts after $24 \mathrm{~h}$ of 1

treatment prevented LPS-induced increased expression of integrin $\beta 3$ and lung tissue autophagy inhibition. Cilengitide treatment also prevented LPS-induced pulmonary fibrosis (Fig. 8b-d), suggesting that integrin $\beta 3$ upregulation mediated LPS-induced lung tissue autophagy inhibition in LPS-induced pulmonary fibrosis.

\section{Discussion}

Although the roles of LPS-induced PI3K-Akt-mTOR pathway activation in lung fibroblast autophagy inhibition and pulmonary fibrosis have been confirmed by our $\mu \mathrm{g} / \mathrm{ml}$ LPS challenge after transfection with Thy-1-OE or empty vector (c). Lung fibroblasts were observed by transmission electron microscopy. White arrows indicate autophagosomes (e), the semiquantification of autophagosomes of per cell were also shown (f). Values are mean $\pm \mathrm{SD}(n=3) . * p<0.05$ vs. control group; $* * p<0.01$ vs. control group; ${ }^{\#} p<0.05$ vs. LPS group; ${ }^{\# \#} p<0.05$ vs. LPS group

previous studies, the underlying mechanisms remain unknown. This study revealed that Thy-1 depletion and integrin $\beta 3$ upregulation mediated PI3K-Akt-mTOR pathway-associated inhibition of lung fibroblast autophagy in LPS-induced pulmonary fibrosis.

Autophagy is an important defense mechanism for cells, and plays a vital role in maintaining homeostasis [22, 23]. The PI3K-Akt pathway targeting mTOR mediates many physiological functions such as cell proliferation, differentiation, migration and apoptosis, and also constitutes an important signaling pathway regulating autophagy [24, 25]. Studies have shown that the PI3K-Akt pathway controlling mTOR expression negatively regulates autophagy in cells 
Fig. 7 Thy-1 overexpression prevents LPS-induced autophagy inhibition and LPSinduced pulmonary fibrosis. Male C57BL/6J mice aged 6-8 weeks ( $n=6$ /group) were treated intratracheally with AAV6-CMV-Thy-1 $\left(5 \times 10^{10}\right.$ $\mathrm{vg}$ /mouse) or vector AAV, as shown in (a). Mice were treated with vector-AAV or Thy- $1^{+}$ -AAV, and Thy-1 mRNA levels were assessed (b). Western blot was performed to detect the expression levels of Thy-1, LC3, P62 (c) and $\alpha$-SMA (d) in the lung tissue. The severity of collagen deposition was measured by assessing hydroxyproline and collagen amounts (e). The severity of pulmonary fibrosis was determined by hematoxylineosin (H\&E) staining; collagen deposition was assessed by Masson's trichrome staining, and $\alpha$-SMA expression in the lung tissue was detected by immunohistochemistry (magnification, $\times 200)(\mathbf{f})$

Values are mean $\pm \operatorname{SD}(n=6)$. $* p<0.05$ vs. control group; $* * p$ $<0.01$ vs. control group; ${ }^{\#} p<$ 0.05 vs. LPS group; ${ }^{\# \#} p<0.05$ vs. LPS group

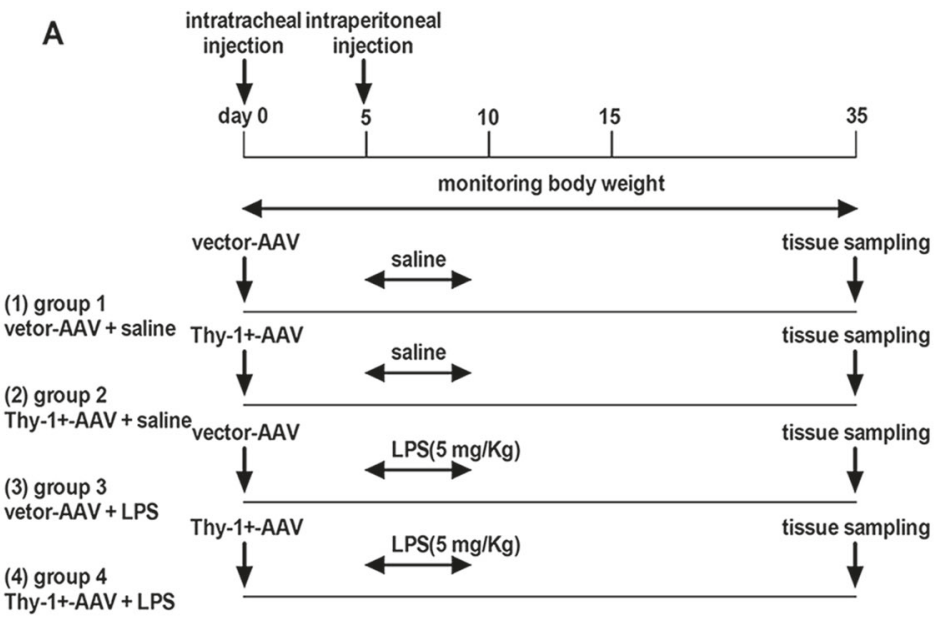

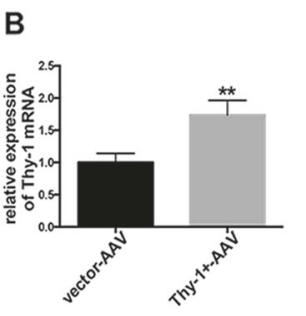

D

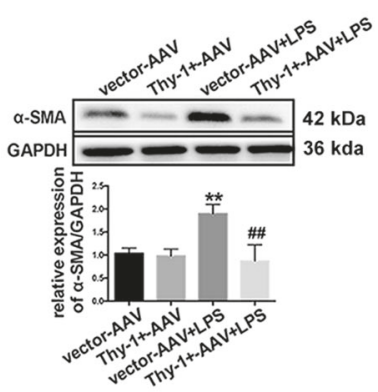

C
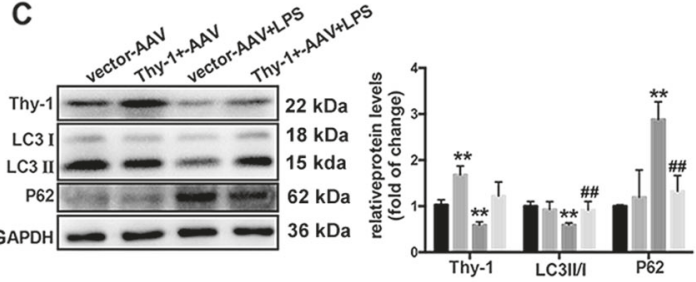

$\mathrm{E}$
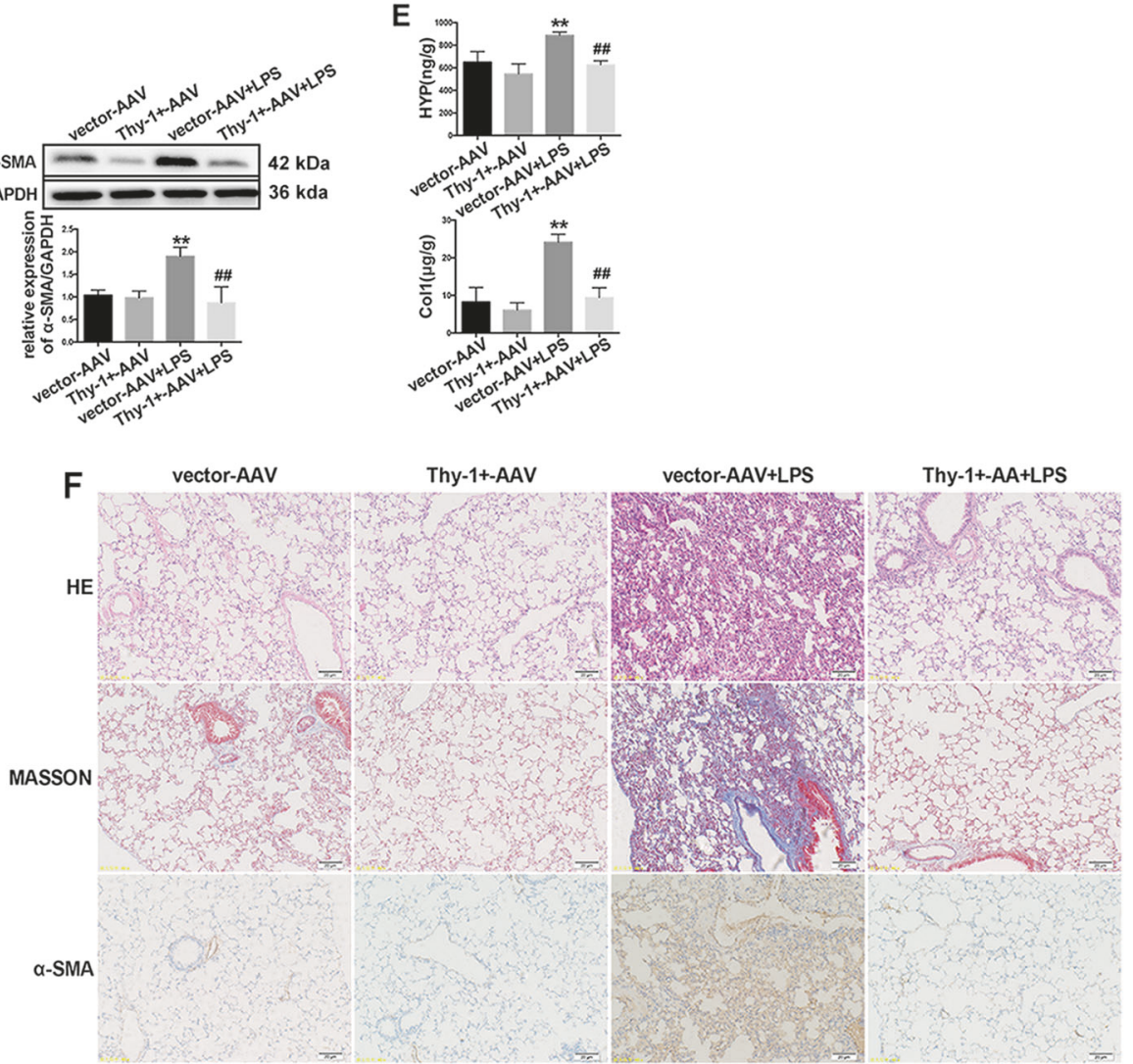

stimulated by factors such as starvation and hypoxia $[26,27]$. Our previous study demonstrated that LPS activates the PI3K-Akt-mTOR pathway and inhibits autophagy in lung fibroblasts [9]. However, the mechanism by which LPS induces PI3K-Akt-mTOR pathway activation remains unclear. 

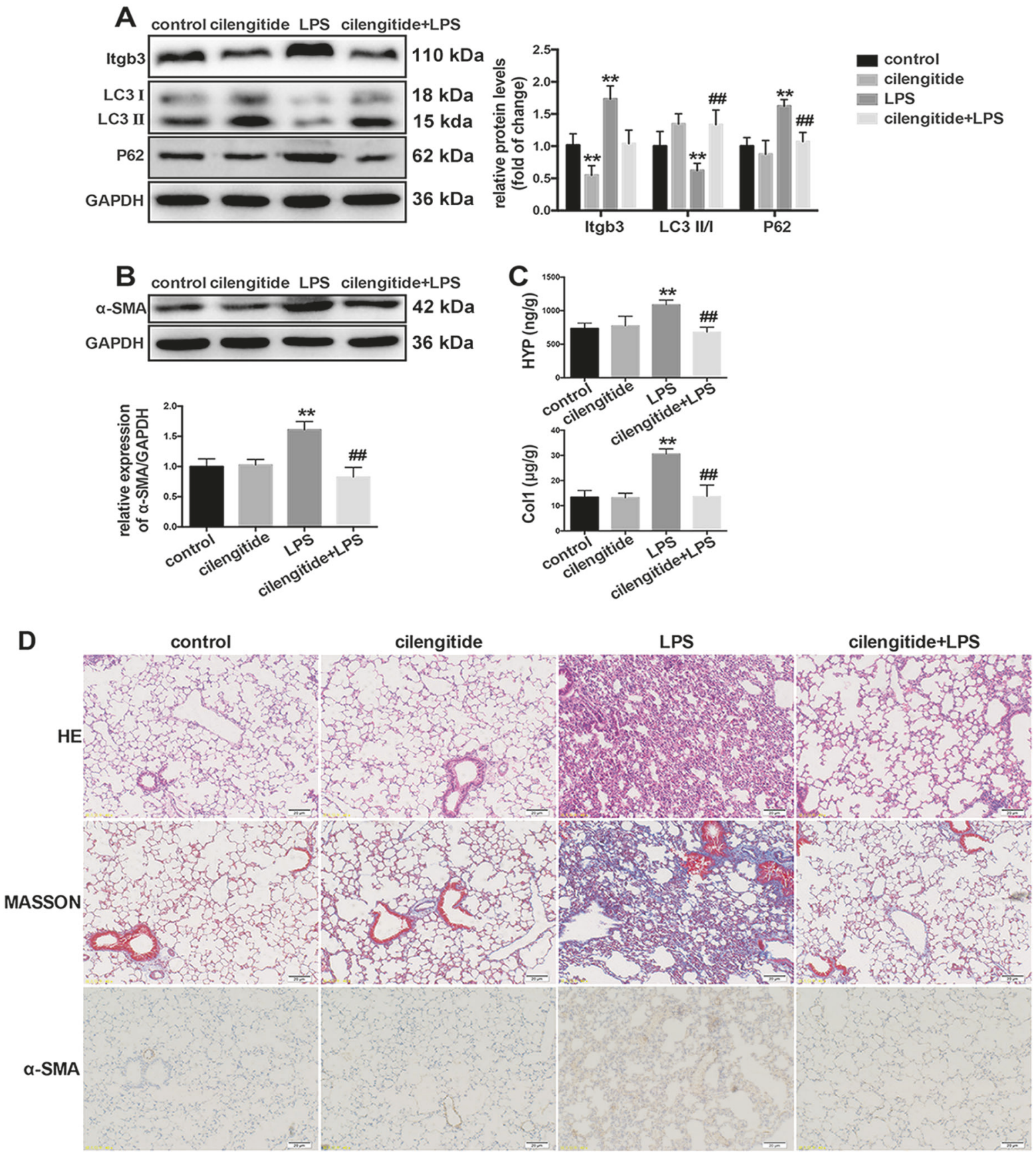

Fig. 8 Integrin $\beta 3$ inhibition prevents LPS-induced autophagy inhibition and LPS-induced pulmonary fibrosis. Male C57BL/6J mice aged $6-8$ weeks ( $n=6 /$ group) were pretreated with cilengitide $(2 \mathrm{mg} / \mathrm{kg})$, followed by LPS $(5 \mathrm{mg} / \mathrm{kg})$ administration for consecutive 5 days. Western blot was performed to detect the expression levels of integrin $\beta 3$, LC3, P62 (a) and $\alpha$-SMA (b) in the lung tissue. The severity of collagen deposition was measured by assessing hydroxyproline and

Thy-1 is a glycosylphosphatidylinositol-linked cell surface glycoprotein expressed on fibroblast subpopulations and various cell types, including thymocytes, $\mathrm{T}$ cells, and nerve cells [28]. As a membrane-bound glycoprotein, Thy-1 is also an important regulator of cell-cell and cell-matrix interactions, activates intracellular signaling pathways, and collagen amounts (c). The severity of pulmonary fibrosis was determined by hematoxylin-eosin (H\&E) staining; collagen deposition was revealed by Masson's trichrome staining, and $\alpha$-SMA expression in the lung tissue was detected by immunohistochemistry (magnification, $\times 200)(\mathbf{d})$. Values are mean $\pm \mathrm{SD}(n=6) . * p<0.05$ vs. control group; ** $p<0.01$ vs. control group; ${ }^{\#} p<0.05$ vs. LPS group; ${ }^{\# \#} p<0.05$ vs. LPS group

affects a variety of cellular functions such as cell proliferation, differentiation, and survival $[29,30]$. Thy- 1 is inversely associated with phenotypic characteristics in fibrosis and considered a "fibrosis suppressor gene" [31]. Several studies have suggested that the absence of Thy- 1 in lung fibroblasts correlates with pulmonary fibrosis [32, 33], 
and our previous study revealed that Thy-1-related lung fibroblast phenotype transformation is essential for LPSinduced lung fibroblast proliferation. Integrin $\beta 3$ is an important adhesion molecule [34] involved in cell proliferation, survival and cancer metastasis. It has been suggested that integrin $\beta 3$ may be a viable anti-fibrotic target in various fibrotic diseases. In bleomycin-induced pulmonary fibrosis, integrin $\beta 3$ expression is particularly enhanced in mesenchymal stromal cells. In normal Thy- $1(+)$ lung fibroblasts, Thy- 1 interacts with integrin $\alpha v \beta 3$ to inhibit collagen-induced cell activation [31, 32]. Meanwhile, studies have confirmed that loss of Thy-1 expression leads to the activation of integrin $\beta 3$ and the downstream FAK/ $\mathrm{PI} 3 \mathrm{~K} / \mathrm{Rac} 1$ signaling pathway $[35,36]$. As shown above, LPS induced Thy- 1 depletion and integrin $\beta 3$ upregulation played an important role in LPS-induced PI3K-Akt-mTOR pathway activation and lung fibroblast autophagy inhibition and pulmonary fibrosis.

According to previous study, the interaction between Thy- 1 and integrin $\beta 3$ can affect the actvation of integrin $\beta 3$ [37]. Referring to a previous research [38], we used Co-IP to analyze the interaction of Thy- 1 and integrin $\beta 3$. Our results revealed a strong interaction between Thy- 1 and integrin $\beta 3$ in lung fibroblasts, which decreased significantly after LPS challenge. Subsequently, we applied flow cytometry to detect PAC-1, a marker of integrin $\beta 3$ activation, in lung fibroblasts [39, 40]. The results showed that LPS challenge led to enhanced activation of integrin $\beta 3$ in lung fibroblasts. integrin $\beta 3$ has been reported to be upstream of the PI3K-Akt-mTOR pathway in various cell types. Because integrin receptors do not possess a catalytic activity, the signaling pathway induced by integrin activation must be transmitted into cells through activation of integrin-associated proteins such as PI3K and FAK. This indicates PI3K-Akt-mTOR signaling or similar pathways may be activated when integrin $\beta 3$ is overexpressed. Studies have shown that integrin $\beta 3$ upregulation inhibits the autophagic process in cardiomyocytes by activating Akt [41], suggesting that the expression status of integrin may affect cell autophagy.

As shown above, genetic or pharmacological inhibition of integrin $\beta 3$ or Thy-1 upregulation prevented LPSinduced PI3K-Akt-mTOR activation and lung fibroblast autophagy inhibition. We also found that Thy-1 overexpression or integrin $\beta 3$ inhibition in vivo could prevent LPS-induced lung tissue autophagy and pulmonary fibrosis, suggesting that early intervention of Thy- 1 and integrin $\beta 3$ in ARDS could block the progression of pulmonary fibrosis, and is expected to improve the cure rate of patients with ARDS-associated respiratory failure, which is important for improving the prognosis of ARDS patients.

Overall, this study demonstrated that depleting Thy-1 and upregulating integrin $\beta 3$ is an important mechanism and potential therapeutic target for PI3K-Akt-mTOR pathwayassociated inhibition of lung fibroblast autophagy in LPSinduced pulmonary fibrosis.

\section{Disclaimer}

The funders had no role in study design, data collection and analysis, decision to publish, or manuscript preparation.

Funding: This study was supported by grants from the National Natural Science Foundation of China (NSFC, No. 81670057 and 81870052) and Training Program Foundation for Distinguished Young Medical Professional from Shanghai Municipal Commission of Health and Family Planning (No. 2018-16).

\section{Compliance with ethical standards}

Conflict of interest The authors declare that they have no conflict of interest.

Publisher's note: Springer Nature remains neutral with regard to jurisdictional claims in published maps and institutional affiliations.

\section{References}

1. Thompson BT, Chambers RC, Liu KD. Acute respiratory distress syndrome. N Engl J Med. 2017;377:1904-5.

2. Armstrong L, Thickett DR, Mansell JP, Billinghurst RC, Poole $\mathrm{AR}$, Millar AB. Changes in collagen turnover in early acute respiratory distress syndrome. Am J Respir Crit Care Med. 1999;160:1910-5.

3. Zinter MS, Delucchi KL, Kong MY, Orwoll BE, Spicer AS, Lim MJ, et al. Early plasma matrix metalloproteinase profiles: a novel pathway in pediatric acute respiratory distress syndrome. Am J Respir Crit Care Med. 2018;199:181-9.

4. Morrison TJ, Jackson MV, Cunningham EK, Kissenpfennig A, McAuley DF, O'Kane CM, et al. Mesenchymal stromal cells modulate macrophages in clinically relevant lung injury models by extracellular vesicle mitochondrial transfer. Am J Respir Crit Care Med. 2017;196:1275-86.

5. Mei SHJ, McCarter SD, Deng Y, Parker CH, Liles WC, Stewart DJ. Prevention of LPS-induced acute lung injury in mice by mesenchymal stem cells overexpressing angiopoietin 1. PLoS Med. 2007;4:e269.

6. Cicko S, Kohler TC, Ayata CK, Muller T, Ehrat N, Meyer A, et al. Extracellular ATP is a danger signal activating P2X7 receptor in a LPS mediated inflammation (ARDS/ALI). Oncotarget. 2018;9:30635-48.

7. Cabrera S, Maciel M, Herrera I, Nava T, Vergara F, Gaxiola M, et al. Essential role for the ATG4B protease and autophagy in bleomycin-induced pulmonary fibrosis. Autophagy. 2015; 11:670-84.

8. Liu H, Fang S, Wang W, Cheng Y, Zhang Y, Liao H, et al. Macrophage-derived MCPIP1 mediates silica-induced pulmonary fibrosis via autophagy. Part Fibre Toxicol. 2016;13:55.

9. Xie T, Xu Q, Wan H, Xing S, Shang C, Gao Y, et al. Lipopolysaccharide promotes lung fibroblast proliferation through autophagy inhibition via activation of the PI3K-Akt-mTOR pathway. Lab Investig J Tech Methods Pathol. 2019;99:625-33. 
10. McCullough KM, Choi D, Guo J, Zimmerman K, Walton J. Epigenetic regulation of Thy-1 gene expression by histone modification is involved in lipopolysaccharide-induced lung fibroblast proliferation. DG, et al. Molecular characterization of Thy 1 expressing fear-inhibiting neurons within the basolateral amygdala. Nat Commun. 2016;7:13149.

11. He Z, Wang X, Deng Y, Li W, Chen Y, Xing S, et al. Epigenetic regulation of Thy-1 gene expression by histone modification is involved in lipopolysaccharide-induced lung fibroblast proliferation. J Cell Mol Med. 2013;17:160-7.

12. Xing S, Nie F, Xu Q, Deng Y, Li W, Yang Z, et al. HDAC is essential for epigenetic regulation of Thy-1 gene expression during LPS/TLR4-mediated proliferation of lung fibroblasts. Lab Investig J Tech Methods Pathol. 2015;95:1105-16.

13. Li W, Xu Q, Deng Y, Yang Z, Xing S, Zhao X, et al. Highmobility group box 1 accelerates lipopolysaccharide-induced lung fibroblast proliferation in vitro: involvement of the NF-kappaB signaling pathway. Lab Investig $\mathrm{J}$ Tech Methods Pathol. 2015;95:635-47.

14. Schmidt M, Gutknecht D, Anastassiadis K, Eckes B, Anderegg U, Saalbach A. Thy-1/beta3 integrin interaction-induced apoptosis of dermal fibroblasts is mediated by up-regulation of fasl expression. J Invest Dermatol. 2016;136:526-9.

15. Whyte CS, Swieringa F, Mastenbroek TG, Lionikiene AS, Lance MD, van der Meijden PEJ, et al. Plasminogen associates with phosphatidylserine-exposing platelets and contributes to thrombus lysis under flow. Blood. 2015;125:2568-78.

16. Hisamoto N, Tsuge A, Pastuhov SI, Shimizu T, Hanafusa H, Matsumoto K. Phosphatidylserine exposure mediated by ABC transporter activates the integrin signaling pathway promoting axon regeneration. Nat Commun. 2018;9:3099.

17. Lu Y-Y, Zhao X-K, Yu L, Qi F, Zhai B, Gao C-Q, et al. Interaction of Src and Alpha-V integrin regulates fibroblast migration and modulates lung fibrosis in a preclinical model of lung fibrosis. Sci Rep. 2017;7:46357.

18. Pechkovsky DV, Scaffidi AK, Hackett TL, Ballard J, Shaheen F, Thompson PJ, et al. Transforming growth factor betal induces alphavbeta3 integrin expression in human lung fibroblasts via a beta3 integrin-, c-Src-, and p38 MAPK-dependent pathway. J Biol Chem. 2008;283:12898-908.

19. Tian B, Lessan K, Kahm J, Kleidon J, Henke C. beta 1 integrin regulates fibroblast viability during collagen matrix contraction through a phosphatidylinositol 3-kinase/Akt/protein kinase B signaling pathway. J Biol Chem. 2002;277:24667-75.

20. Dong W-W, Zhang Y-Q, Zhu X-Y, Mao Y-F, Sun X-J, Liu Y-J, et al. Protective effects of hydrogen-rich saline against lipopolysaccharide-induced alveolar epithelial-to-mesenchymal transition and pulmonary fibrosis. Med Sci Monit Int Med J Exp Clin Res. 2017;23:2357-64.

21. Dehghani S, Rasoulianboroujeni M, Ghasemi H, Keshel SH, Nozarian Z, Hashemian MN, et al. 3D-Printed membrane as an alternative to amniotic membrane for ocular surface/conjunctival defect reconstruction: an in vitro $\&$ in vivo study. Biomaterials. 2018;174:95-112.

22. Cadwell K. Crosstalk between autophagy and inflammatory signalling pathways: balancing defence and homeostasis. Nat Rev Immunol. 2016;16:661-75.

23. Hansen M, Rubinsztein DC, Walker DW. Autophagy as a promoter of longevity: insights from model organisms. Nat Rev Mol Cell Biol. 2018;19:579-93.

24. Chang F, Lee JT, Navolanic PM, Steelman LS, Shelton JG, Blalock WL, et al. Involvement of PI3K/Akt pathway in cell cycle progression, apoptosis, and neoplastic transformation: a target for cancer chemotherapy. Leukemia. 2003;17:590-603.
25. Chen N, Debnath J. IkappaB kinase complex (IKK) triggers detachment-induced autophagy in mammary epithelial cells independently of the PI3K-AKT-MTORC1 pathway. Autophagy. 2013;9:1214-27.

26. Lamoureux F, Zoubeidi A. Dual inhibition of autophagy and the AKT pathway in prostate cancer. Autophagy. 2013;9:1119-20.

27. Saiki S, Sasazawa Y, Imamichi Y, Kawajiri S, Fujimaki T, Tanida I, et al. Caffeine induces apoptosis by enhancement of autophagy via PI3K/Akt/mTOR/p70S6K inhibition. Autophagy. 2011;7:176-87.

28. Neveu WA, Mills ST, Staitieh BS, Sueblinvong V. TGF-beta1 epigenetically modifies Thy-1 expression in primary lung fibroblasts. Am J Physiol Cell Physiol. 2015;309:C616-26.

29. Yousefi-Rad N, Shokrgozar MA, Behdani M, Moradi-Kalbolandi S, Motamedi-Rad M, Habibi-Anbouhi M. Antigenic assessment of a recombinant human CD90 protein expressed in prokaryotic expression system. Protein Expr Purif. 2015;116:139-43.

30. Herrera-Molina R, Valdivia A, Kong M, Alvarez A, Cardenas A, Quest AFG, et al. Thy-1-interacting molecules and cellular signaling in cis and trans. Int Rev Cell Mol Biol. 2013;305:163-216.

31. Cohen PY, Breuer R, Wallach-Dayan SB. A profibrotic phenotype in naive and in fibrotic lung myofibroblasts is governed by modulations in thy- 1 expression and activation. Mediators Inflamm. 2018;2018:4638437.

32. Zhou W-Q, Wang P, Shao Q-P, Wang J. Lipopolysaccharide promotes pulmonary fibrosis in acute respiratory distress syndrome (ARDS) via lincRNA-p21 induced inhibition of Thy-1 expression. Mol Cell Biochem. 2016;419:19-28.

33. Sanders YY, Pardo A, Selman M, Nuovo GJ, Tollefsbol TO, Siegal GP, et al. Thy-1 promoter hypermethylation: a novel epigenetic pathogenic mechanism in pulmonary fibrosis. Am J Respir Cell Mol Biol. 2008;39:610-8.

34. Zhou Y, Hagood JS, Lu B, Merryman WD, Murphy-Ullrich JE. Thy-1-integrin alphav beta5 interactions inhibit lung fibroblast contraction-induced latent transforming growth factor-beta1 activation and myofibroblast differentiation. $J$ Biol Chem. 2010;285:22382-93.

35. Burgos-Bravo F, Figueroa NL, Casanova-Morales N, Quest AFG, Wilson CAM, Leyton L. Single-molecule measurements of the effect of force on Thy-1/alphavbeta3-integrin interaction using nonpurified proteins. Mol Biol Cell. 2018;29:326-38.

36. Qiu W, Zhang Y, Liu X, Zhou J, Li Y, Zhou Y, et al. Sublytic C5b-9 complexes induce proliferative changes of glomerular mesangial cells in rat Thy-1 nephritis through TRAF6-mediated PI3K-dependent Akt1 activation. J Pathol. 2012;226:619-32.

37. Fiore VF, Strane PW, Bryksin AV, White ES, Hagood JS, Barker TH. Conformational coupling of integrin and Thy-1 regulates Fyn priming and fibroblast mechanotransduction. J Cell Biol. 2015;211:173-90.

38. Pankow S, Bamberger C, Calzolari D, Bamberger A, Yates JR 3rd. Deep interactome profiling of membrane proteins by co-interacting protein identification technology. Nat Protoc. 2016;11:2515-28.

39. Tadokoro S, Nakazawa T, Kamae T, Kiyomizu K, Kashiwagi H, Honda $\mathrm{S}$, et al. A potential role for alpha-actinin in inside-out alphaIIbbeta3 signaling. Blood. 2011;117:250-8.

40. Ghevaert C, Salsmann A, Watkins NA, Schaffner-Reckinger E, Rankin A, Garner SF, et al. A nonsynonymous SNP in the ITGB3 gene disrupts the conserved membrane-proximal cytoplasmic salt bridge in the alphaIIbbeta3 integrin and cosegregates dominantly with abnormal proplatelet formation and macrothrombocytopenia. Blood. 2008;111:3407-14.

41. Zhu Y, Li L, Gong S, Yu Y, Dai H, Cai G, et al. ss3-integrin inhibits lipopolysaccharide-induced autophagy in cardiomyocytes via the Akt signaling pathway. Cardiology. 2015;130:249-59. 